Théologiques

Théologiques

\title{
La question des augures à Rome : L'éthique du devenir incertain
}

\section{Christian St-Germain}

Volume 8, numéro 1, printemps 2000

La superstition

URI : https://id.erudit.org/iderudit/005028ar

DOI : https://doi.org/10.7202/005028ar

Aller au sommaire du numéro

\section{Éditeur(s)}

Faculté de théologie de l'Université de Montréal

ISSN

1188-7109 (imprimé)

1492-1413 (numérique)

Découvrir la revue

Citer cet article

St-Germain, C. (2000). La question des augures à Rome : L'éthique du devenir incertain. Théologiques, 8(1), 85-104. https://doi.org/10.7202/005028ar
Résumé de l'article

Dans ce bref essai, nous cherchons à surprendre la poésie d'une folieclassificatoire qui se révèle à travers les subdivisions spécialisées des" métiers d'avenir " d'obédiences jupitériennes, des collèges de devins del'ancienne Rome : Augures, Haruspices, Flamines, toutes espèces detechniciens des foudres et des viscères d'oiseaux affichant une inégalablecomponction dans l'exercice de leur art.L'acte divinatoire vise à donner du sens et surtout à en trouver dansl'univers, partie liée avec le phénomène religieux. Toute une herméneutiquesavante de l'inopiné, de l'aléatoire ou de l'impondérable vient réduirel'inconnu à la certitude de signes qui ne sauraient mentir. Il en va tout autantdans la religion que dans la divination du maniement d'un transfert, de laproposition à un sujet angoissé d’un savoir supposé certain, quant à l'avenir. 


\section{La question des augures à Rome L'éthique du devenir incertain}

Christian ST-GermaIN

Rattaché au vice rectorat à la formation Université du Québec à Montréal

"Il s'agit de faire dire aux dieux, à Dieu, à ce qui tient la place de l'Autre fabuleux comment l'bumanité doit être divisée, en quel sens. Inaugural, ce terme qui nous vient de la science des augures — la branche la plus antique du droit romain - implique la reconnaissance d'un lieu de la certitude, la zone divine où ça sait ». ${ }^{1}$

Pierre Legendre

Un dieu qui ne menacerait, ni ne rassurerait, en quoi serait-il un dieu?

Les amoureux connaissent bien cette expérience naïve des réminiscences d'un parfum, d'un vêtement, portés lors de la première rencontre avec l'aimée, de la chanson entendue au réveil et qui répond étrangement à la question inquiète posée en silence au moment de s'endormir. Nous accumulons sans cesse les talismans de cette candeur contre les solitudes blafardes. En collectionnant ces coïncidences, notre vie s'écoule dans leur chapelet. Dans l'ancienne Rome, l'omen ${ }^{2}$, le fait justement d'intercepter une parole qui ne nous est pas adressée mais susceptible de trouver une application personnelle est

1. Pierre LEGENDRE, Leçons VII Le désir politique de Dieu. Étude sur les montages de l'État et du Droit, Paris, Arthème Fayard, 1988, p. 160.

2. "Faut-il rappeler l'exemple fameux de l'omen donné à Crassus, partant en expédition contre les Parthes, par un marchand de figue criant : "Cauneas" (sous-entendu ficos) c'est-à-dire (achetez) mes figues de Caunos, ville de Carie. Mais c'était là un avertissement que le général romain ne comprit pas car le cri devait s'entendre Caue ne eas, prends garde, n'y va pas ". Raymond BLOCH, La divination dans l'Antiquité, Paris, PUF, 1984, p. 82. 
compris comme un signe, une réponse plausible des dieux à ce souci de soi minuscule dans l'univers. ${ }^{3}$

Dans ce bref essai, nous ne voudrions pas cautionner les pratiques oraculaires modernes ou réhabiliter la crédulité du commun ni cependant non plus, ne voir dans la divination, qu'un reliquat animiste. Nous préférons surprendre la poésie d'une folie classificatoire à l'occasion de la rationalité jurisprudentielle visible dans les subdivisions spécialisées des "métiers d'avenir » d'obédiences jupitériennes, des collèges de devins de l'ancienne Rome : Augures ${ }^{4}$, Haruspices ${ }^{5}$,

3. "L'omen est, à proprement parler, le présage entendu et la divination ominale a toujours eu une grande importance à Rome. Toute phrase prononcée par un tiers dans une intention qui lui est propre mais pouvant littéralement s'appliquer aux préoccupations et à la conduite du sujet intéressé constitue un présage pouvant inspirer crainte ou confiance. Les omina portent en eux l'avenir mais il s'agit d'un avenir proche ou immédiat et ce sont des avertissements envoyés par les dieux aux hommes pour les confirmer dans leurs entreprises ou bien au contraire les en détourner ". Raymond BLOCH, "La religion romaine ", Histoire des Religions 1. Encyclopédie de la Pléiade, Paris, Gallimard, 1970, p. 898.

4. "Outres les consultations des auspices, les augures procèdent à l'inauguration rituelle de telle ville, de certains temples, de locaux et des lieux consacrés aux affaires publiques (...) Ils avaient leurs " actes » et leurs « Commentaires » comme les Pontifes; et leurs livres de doctrine, où tout un vocabulaire technique spécifiait maintes minuties d'observation ". Jean BAYET, Histoire politique et psychologique de la religion romain, Paris, Payot, 1969, p. 104.

5. Les haruspices conjuguent à la science de l'observation des foies d'oiseaux (l'extispicine) celle non moins intrigante, de la science des foudres. Ils sont originaires d'Etrurie et utilisent d'abord des foies d'oiseaux comme maquette du plan d'une ville et des événements qui devaient s'y produire. Quant à leur herméneutique des foudres, Raymond Bloch écrit : "Seul, en vérité, un prêtre aux vastes connaissances pouvait déterminer d'après la couleur de la foudre, l'heure à laquelle elle apparaissait, suivant l'objet ou l'être frappé, le tonnerre qui l'accompagnait et suivant tous les modes de son effet matériel. (...) Quantité de distinctions internes intervenaient qui prêtaient à la doctrine étrusque l'aspect d'une casuistique impénétrable au noninitié. (...) Mais l'activité de l'haruspice ne s'arrêtait pas là. Il fallait qu'il procède aussi à l'expiation des foudres. (...) Il procède à l'ensevelissement des foudres (fulmen condere), il enfouit toutes les traces matérielles de son passage et sacrifie les brebis (bidentes) aux dieux, d'où le nom, à l'époque, 
Flamines ${ }^{6}$, toutes espèces de techniciens des foudres et des viscères d'oiseaux $^{7}$ affichant une inégalable componction dans l'exercice de leur art. $^{8}$

L'on imagine d'ailleurs à Rome, la procession étrange de ces poissons clowns, de ces vieilles murènes dépenaillées, affamées de présages, arborant l'apex ${ }^{9}$ et maniant le lituus. ${ }^{10}$ Seule peut-être à notre époque, une réunion de sémioticiens devisant sur un conte de Perrault pourrait se rapprocher de cette superbe dérisoire. Plus sérieusement,

romaine, de bidentalia donné à ces puits à foudre ». Raymond BLOCH, La divination dans l'Antiquité, Paris, PUF, 1984 p. 66.

6. Le Flamine de Jupiter est intéressant dans la mesure où il est l'incarnation de la divinité majeure de Rome. Il est le prêtre "le plus fameux et le plus vénérable de la cité ». Il est enraciné à Rome dit John Scheid : "Il ne pouvait pas passer une seule nuit hors de Rome sans commettre une impiété, et il ne pouvait découcher trois nuits consécutives de son lit, dont les pieds, enduits d'une légère couche de boue, traduisaient une communication physique permanente avec le sol de Rome ». Il est en quelque sorte, à la fois le paratonnerre et la figure de la foudre jupitérienne qui descend dans le sol. « (...) le flammeum était de couleur jaune rouge, la couleur du feu et de la foudre jovienne » «(...) il lui est interdit de voir et de toucher un cadavre, de pénétrer dans l'enceinte d'un bûcher funèbre (...) de consommer des fèves en raison de leur connotation funeste ». Il ne prête aucun serment et ne se lie d'aucune manière car il est l'incarnation du droit : souverain, libre et déliant : "Il ne peut ni toucher ni nommer le lierre puisque le lierre lie tout ce qu'il agrippe ». John SCHEID, «Le flamine de Jupiter, les Vestales et le général triomphant. Variations romaines sur le thème de la figuration des dieux ", dans Charles MALAMOUD (dir.), Corps des dieux, Paris, Gallimard, 1986, p. 213-229, p. 216-218.

7. Tel un instantané ou une image polaroid : "La consécration de la victime était censée produire en ses organes une sorte de projection immédiate du monde telle que le pouvait saisir la vision sans erreur des dieux. Les haruspices interprétaient d'après l'état du flux sanguin, la couleur, la conformation de telle ou telle partie du foie.» Jean BAYET, Histoire politique et psychologique de la religion romaine, Paris, Payot, 1969, p. 58.

8. Déjà Caton s'interrogeait sur la sincérité des interprètes en ces termes: "Peut-être les Augures ou les Haruspices, qui connaissaient, eux, de l'intérieur, la vanité de leur sciences, riaient-ils entre eux. » Danielle PORTE, Les donneurs de sacré. Le prêtre à Rome, Paris, Éditions les Belles Lettres, 1989, p. 8.

9. Partie supérieure du bonnet du flamine, laquelle se compose d'une petite baguette entourée de laine.

10. Il s'agit d'un bâton courbe, l'outil par excellence du sacerdoce augural. 
la fragilité d'un texte sur un sujet aussi complexe demeure celle d'avoir été écrit trop rapidement pour tirer au clair toutes les implications sociales et religieuses de ce partage du pouvoir autour des solennités de cette lecture. À l'égard de la divination romaine, le profane à l'impression de se trouver auprès d'une religion dont l'unique livre révélé tiendrait à un Lévitique morcelé, à un imbroglio de prescriptions composites et autonomes.

Il en va cependant des mécanismes subtils du montage du complexe politico-religieux romain dans son affirmation d'universalité. ${ }^{11}$ Qui pourrait prétendre expliquer aujourd'hui même, parfaitement, la division des prérogatives entre le directeur de la banque fédérale, le président des États-Unis et le sénat dans l'équilibre interne de la politique monétaire ou extérieure américaine? L'établissement rétrospectif des liens entre chacun des herméneutes antiques s'avère donc une entreprise essentiellement conjecturale.

\section{Le fonctionnement gémellaire : religion-superstition}

Toute une politique du scrupule se trouve dans cette observance des signes et dans ces travaux d'enfants cruels. Et dans ce contexte particulier, la superstition n'est qu'une excroissance de la religion elle-même, une doublure décousue à la frange déjà exagérée des interdits : "Bon nombre de modernes, après les Anciens, s’y sont résignés : superstitio désignerait "ce qui est en sus ", " ce qui dépasse » (fâcheusement) les prescriptions de la religion officielle $»{ }^{12}$ Dans cette proximité étymologique, la superstition se ramène à une forme d'obséquiosité rituelle, si l'on considère l'expression de la religio latine comme " une hésitation qui retient, un scrupule qui empêche, et non un sentiment qui dirige vers une action, ou qui incite à pratiquer un culte $» .{ }^{13}$ Le religere concerne l'attention soutenue, l'extrême minutie accordée aux actions posées en

11. Ce remaniement du cadre du pouvoir antique, de l'autorité faisait écrire à Freud que : " notre vieux Monde est régi par l'Autorité, comme le Nouveau par le Dollar. " Pierre LEGENDRE, L'amour du censeur. Essai sur l'ordre dogmatique, Paris, Seuil, 1974, p. 30.

12. Huguette FUGIER, Recherches sur l'expression du sacré dans la langue latine, Paris, Les Belles Lettres, 1963, p. 175.

13. Émile BENVENISTE, Le vocabulaire des institutions indo-européennes 2. pouvoir, droit, religion, Paris, Éditions de Minuit, 1969, p. 270. 
vue de conserver la paix des dieux (Pax Deorum). Ce qui s'oppose à la religion latine c'est le neglegere ${ }^{14}$ et non quelques formes de négations intellectuelles du fait des interventions divines nécessaires pour assurer la stabilité du monde.

La superstition n'est surtout pas opposée à la religion ni n'a de sens péjoratif mais naît par implication nécessaire de celle-ci, de son souci de l'observance du détail. La divination reste le moyen par lequel les divinités font connaître l'étendue de leur mansuétude providentielle aux citoyens romains. Même Cicéron, tout réservé et critique qu'il a pu être à l'égard des mantiques, n'hésitera pas à reconnaître, dans son traité intitulé De la nature des dieux, que :

Les haruspices discernent et les augures prévoient bien des choses; les oracles, les prophéties, les songes, les prodiges en dévoilent beaucoup ; et grâce aux connaissances ainsi acquises, bien souvent des décisions sont prises, des avantages créés et aussi des dangers évités. Ce pouvoir, qu'il soit acquis par l'art ou inné, a été certainement donné à l'homme par les dieux en vue de la connaissance de l'avenir. (...) Ce n'est pas seulement sur le genre humain pris en général, c'est sur chaque individu que veille la providence des dieux immortels. ${ }^{15}$

Il suffit de prêter attention aux signes et l'oreille aux voix pour constater que dehors le monde est gouverné. La sagesse consiste à ne pas se mettre en travers des chemins que prennent les dieux pour protéger Rome de ses ennemis, à ne pas interrompre le cours de cette invisible sollicitude. C'est que l'attitude religieuse par excellence tient à une forme d'inhibition obstinée quant au fait d'agir et quant à l'obligation de réunir les précautions nécessaires à tout éventuel commencement.

\section{Prière de ne pas déranger}

Dans le cours des actions humaines, il importe de ne rien commettre qui indisposerait les dieux ou dérangerait en quelque manière l'ordre établi : d'où ce délire systématisé d'observations augurales, de prises de signes, d'écoute des avertissements du ciel. À l'inverse de cette vigilance, élevée au rang de piété, l’impiété elle, ne consiste pas

14. Pierre BOYANCÉ, Études sur la religion romaine, Rome, Collection de l'École française de Rome, 1972, p. 32.

15. CICÉRON, " De la nature des dieux ", dans : Émile BRÉHIER (dir.) : Les Stoïciens, Paris, Gallimard, Éditions de la Pléiade, 1962, p. 467. 
tant dans la commission d'une faute morale que dans une erreur procédurale, un accident par inadvertance ou encore la négligence d'un officiant. Comme si l'expression religieuse se confondait avec les exigences extatiques d'un droit administratif à dimension cosmique. L'historien John Scheid écrit à ce propos que :

Est impie et inexpiable l'individu qui viole délibérément les prescriptions rituelles (....). L'exemple le plus courant, quotidien presque, d'une infraction est celui d'une faute commise durant la célébration du culte ou, plus généralement, des fêtes. Dans ce cas la faute consiste dans une erreur rituelle, un oubli, et peut d'ailleurs être signalé par un prodige. Une fois cette infraction constatée, il suffit de répéter totalement ou en partie (ce qui se dit : instaurare) la cérémonie viciée, pour que l'effet religieux de celle-ci soit entier. (...) Une fois que la faute a été constatée ou annoncée, tout retard dans la rémédiation est fatal et transforme l'imprudence en impiété. ${ }^{16}$

Cette inquiétude quant au formalisme de validité d'une action ressemble, au plan individuel cette fois, au souci de l'obsessif qui s'oblige à reprendre du début toutes ces pratiques compulsives dès qu'un geste n'a pas été adéquatement posé.

\section{Profession : devin}

À Rome, le devin n'obtient pas de visions de l'avenir par l'entrée dans une transe écumeuse ou par le tremblement épileptique mais bien plutôt en procédant à une collection méthodique des indices probants au terme d'un examen érudit des signes. Il est dans le sens le plus noble, un "gestionnaire " de l'obsession cadastrale romaine s'assurant de maintenir l'ordo rerum ${ }^{17}$ intact, préservant l'ensemble de la

16. John SCHEID, Religion et piété à Rome, Paris, Éditions de la Découverte, 1985, p. 23.

17. L'Ordo rerum désigne : «(...) la disposition des éléments de l'univers (et aussi des institutions), telle que les Dieux l'ont conçue et établie. La nature de la religion consiste à la préserver contre toute atteinte : les éclipses, les jumeaux, les albinos, les prodiges (....). Une interdiction violée amène des catastrophes, inondations ou épidémies, si le prêtre n'est pas là pour indiquer à temps les moyens de réparer l'injure, c'est-à-dire d'expier l'anomalie, la faute, l'erreur, le crime, le malheur (c'est ici la même chose), afin de restaurer la régularité entamée ". Roger CAILLOIS, Case d'un échiquier, Paris, Gallimard, 1970, p. 24. 
communauté des conséquences naturelles ou météorologiques de la commission de sacrilèges.

Le travail divinatoire s'insère le plus souvent dans le respect strict des limites du sol sacré, au cœur d'un effort de localisation garant de la validité de la lecture. Rien n'est d'ailleurs commencé sans l'intervention de l'Augure, scrupuleux dessinateur de marelles, bien involontaire ancêtre du contrôleur aérien. Danielle Porte constate que :

Il est de fait que la dignité d'Augure apparaît à Rome sinon comme l'une des plus prestigieuses, du moins comme celle qui donne à qui la revêt les clefs du pouvoir. Avant de passer à l'exécution de tout acte public, on doit obtenir la sanction divine sur la décision qu'on pense appliquer. Avant de livrer aux utilisateurs un temple récemment bâti, une ville, une colonie nouvelle, on doit les inaugurer. ${ }^{18}$

La pratique du devin commande avant tout de rejouer le geste souverain d'un découpage originaire. Elle implique la connaissance d'une suite d'opérations topiques de bornage, d'inscriptions de bordures, de franges et de régions du ciel ${ }^{19}$ afin d'opérer une lecture exacte de ce que l'on tente de démêler à travers l'écheveau des banalités, des prodiges ou encore des choses muettes à considérer. C'est que la prise des auspices consiste en une reviviscence de la fondation de Rome. Tout dans le geste de lecture ouvre sur le respect d'un tracé, d'une lisière sacro-sainte (pomoerium), ligne de tous les partages subséquents, que l'on ne saurait purger de son hypothèque ancestrale et, grâce à laquelle, s'oriente ce qu'il advient de la ville et de ses habitants,

18. Danielle PORTE, Les donneurs de sacré. Le prêtre à Rome, Paris, Éditions les Belles Lettres, 1989, p. 151.

19. Comme le note Ernst Cassirer : "La sacralisation commence lorsqu'on dégage, de la totalité de l'espace, une région particulière, qui est distinguée des autres, qui est pour ainsi dire clôturée par le sentiment religieux. Cette notion de sacralisation religieuse, qui se présente aussi comme une division de l'espace, s'est concrétisée linguistiquement dans l'expression templum (...). À partir de cette division originaire de nature purement spatiale se déploie tout le système de la " théologie » romaine. Lorsque l'augure observait le ciel pour y lire des signes de l'action terrestre, il commençait toujours cette opération en divisant le ciel en plusieurs quartiers ». Ernst CASSIRER, La philosophie des formes symboliques. La pensée mythique, T. 2, Paris, Éditions de Minuit, 1974, p. 128. 
en fait, le destin historique des habitants du site. L'historien Raymond Bloch rappelle à ce propos que :

Romulus détermine le tracé des murs de la future Rome en creusant avec le soc d'une charrue un sillon autour du Palatin. L'attelage est composé d'une vache et d'un boeuf blanc comme neige. Les citoyens suivant ce sillon jettent le fondement de la muraille. Ainsi fut déterminé le Pomoerium originel de l'Urbs, c'est-à-dire la zone sacro-sainte et taboue qui entoura d'une frontière efficace et protégée par la divinité le corps même de la ville. ${ }^{20}$

Il importe au devin d'épouser - comme en s'appuyant sur un hypothétique décalque - ce qui paraît être la consécration de l'espace romain par le dieu Jupiter. D'où une attention particulière portée à toutes les marques de ponctuations célestes : par la consultation du vol, des entrailles d'oiseaux mais aussi des pierres de foudre, qu'envoie le dieu pour corriger, informer d'une anomalie ou d'une irrégularité par rapport à la disposition d'un plan céleste ou des lignes de division de cet arpentage initial. L'ordre politique et social est ainsi établi sur le respect de la division augurale. Le philosophe Ernst Cassirer observait, dans sa Philosophie des formes symboliques que :

L'arpentage, cet acte fondamental de « limitation » qui seul produit, au sens juridique et religieux, une propriété stable, se rattache toujours à l'organisation sacrale de l'espace. Dans les écrits des agrimensores romains, c'est-à-dire des arpenteurs, l'introduction de la limitatio est attribuée à Jupiter, et immédiatement rattachée à la création du monde. Tout se passe comme si la stricte division qui règne dans l'univers était ainsi transposée sur la terre et dans toutes les choses terrestres. ${ }^{21}$

C'est cette clôturation foncière de tout l'espace dans l'expérience de la limite statique qui, paradoxalement, met la pensée religieuse romaine en mouvement, l'oblige à se doter de spécialistes religieux (des faiseurs de liens dans le respect des limites) des passages, des ponts, à des dérogations ou à des transgressions autorisées. La limite et le tracé sont investis par une divinité tutélaire, tels les " cases d'un échiquier » pour reprendre le titre d'un ouvrage magnifique de Roger Caillois et traverser ces espaces commande une déférence religieuse à

20. Raymond BLOCH, Les origines de Rome, Paris, PUF, 1990, p. 14.

21. Ernst CASSIRER, La philosophie des formes symboliques. La pensée mythique, T. 2, Paris, Éditions de Minuit, 1974, p. 128. 
l'endroit de la limite franchie ${ }^{22}$ entre ces divins lotissements. Il n'est pas permis de prendre quelque liberté à l'égard du déplacement de ces divisions sans s'assurer d'apaiser préventivement le titulaire du fond.

Il y a ainsi une co-appartenance de la notion de sacré avec celle de la propriété domaniale. Devins et pontifes consultent ponctuellement les dieux pour éviter l'irritation des seuils - accorder symboliquement les droits de passage et les servitudes — sur toutes ces invisibles juridictions. Le droit et la religion romaine se tiennent réciproquement dans un rapport d'indivision intellectuelle, naissent de cette gémellité féconde.

\section{Le sacré comme système de limites}

Une politique exquise à l'égard de la liminarité se développe ainsi pour opérer judicieusement le départage des lignes de force et éventuellement s'assurer que se cicatrisent sans heurt les lèvres disjointes — par la construction d'un pont par exemple - des rives. ${ }^{23}$ Toute action qui contrevient à une disposition naturelle statique du paysage doit d'abord être permise et ensuite faite selon les règles précises. ${ }^{24}$ Évidemment, ces

22. "La ville, mais aussi le compituum et la maison, non seulement le territoire agricole, mais chaque champ labouré, chaque vigne, non seulement la maison dans sa totalité, mais chaque pièce, ont leur dieu propre. On reconnaît la divinité par ses interventions et par ce qui l'environne. C'est pourquoi chaque esprit qui est enfermé dans un espace acquiert une individualité et un nom particulier, par lequel les hommes peuvent l'invoquer ». Ernst CASSIRER, La philosophie des formes symboliques. La pensée mythique, T. 2, Paris, Éditions de Minuit, 1974, p. 128.

23. Cette tâche est de la compétence exclusive du pontifex maximus à Rome. Comme le note Roger Caillois : "Bâtir un pont est un subterfuge sacrilège qui, comme tel, compromet l'ordre du monde et qui ne saurait qu'attirer un terrible châtiment sur son auteur, sa famille, sa nation. (...) De toute façon, par son essence, le pont porte atteinte à une secrète et inextricable économie, où il n'est rien qui ne se tienne et ne se compense. (...) Je remarque enfin sans ironie que la plus haute autorité morale et religieuse du siècle porte le titre de Souverain Pontonnier, sans que personne ne songe aujourd'hui à l'obscure magie qui en explique le mystère ". Roger CAILLOIS, Case d'un échiquier, Paris, Gallimard, 1970, p. 26-27.

24. Un peu comme le droit moderne, en matière d'environnement commande, lorsqu'on le fait respecter, une étude d'impacts et l'émission par l'autorité compétente d'un permis. 
précautions n'obéissent pas à des considérations utiles. L'historienne des religions Danielle Porte souligne que :

L'espace s'appelait templum, de temno = "couper ", et il va sans dire que le même mot désignera le bâtiment terrestre construit sur un espace orienté défini de la même façon par une prise d'auspices. (...) Deux équipes de spécialistes, les Augures et les Haruspices, exerçaient leurs compétences en ce domaine, les uns se consacrant à l'examen des signes célestes, les autres à celui des animaux sacrifiés. L'Antiquité faisait volontiers dériver augurium et auspicium du mot auis, l'oiseau, suivi de gerere ou de spicere "s'occuper de " ou "observer", voyant dans l'auspice une simple manifestation céleste, et dans l'augure l'analyse humaine de ce prodige, et l'annonce des conséquences prévisibles. L'Haruspice est, lui, un " inspecteur d'entrailles » (Haru + spicere). ${ }^{25}$

Dans ce contexte ancien, le monde apparaît comme quadrillé, traversé par une écriture en filigrane, - indiquant de lui-même, à chacun, pour peu qu'il s'y arrête - une direction favorable à déceler. La science divinatoire se développe inextricablement liée à la piété et à la religion. Ses préposés savants à la collection des indices - les Augures $^{26}$ - interviennent pour assurer des correspondances, soulever la nasse des intersignes. ${ }^{27}$ Ils établissent alors, selon un principe de causalité mystérieux, les interconnexions significatives entre une liste préétablie de présages tenue dans leurs livres et reconnue et les événements les plus susceptibles d'advenir. ${ }^{28}$

25. Danielle PORTE, Les donneurs de sacré. Le prêtre à Rome, Paris, Éditions les Belles Lettres, 1989, p. 154-155.

26. "Augurium (...) désigne pour un devin, le don mantique en général (de même qu'Apollon est augur). Mais le plus souvent augurium accompagne chez Virgile des signes qui marquent le commencement de quelque chose ». Pierre BOYANCÉ, La religion de Virgile, Paris, PUF, 1963, p. 104.

27. Intersigne : Lien mystérieux que l'imagination se plaît à établir entre deux faits qui se produisent au même moment, souvent à de grandes distances l'un de l'autre, et dont l'un est considéré comme le pronostic de l'autre ; ainsi le portrait d'une personne aimée se détache de la muraille, et l'on apprend qu'au même moment cette personne est morte; c'est une autre forme de la seconde vue. (E. Littré).

28. Dans les cas de la survenance de prodiges les plus inquiétants l'on consulte les Livres Sibyllins. "Ce recueil sacré passait pour contenir les secrets d'où dépendaient la fortune et la vie même de Rome. Il était placé sous 


\section{Prévisions et procédures}

Le pouvoir paraît donc inséparable à Rome d'une certaine apparence de mainmise sur les événements futurs. Il procède plus exactement du présupposé selon lequel la prévision de l'avenir est non seulement possible mais que cet avenir s'annonce déjà de la plus étrange manière ; qu'il se donne à voir ou même à lire, à travers des indices faméliques ou spectaculaires. ${ }^{29} \mathrm{La}$ connaissance des règles de cette grammaire des liens insolites ou la possession de cette "science diagonale " et de ces " cohérences aventureuses " ${ }^{30}$ confère derechef à son détenteur une situation sociale privilégiée.

L'exercice de prédiction repose sur le principe que chaque suite d'événements défavorables - ici pour la ville romaine mais souvent dans la vie des individus- est précédée par un symptôme, un prodige ${ }^{31}$ qui, bien que sans rapport immédiat ou évident avec l'enchaînement qu'il transporte à sa suite, commande d'être considéré intrinsèquement - en raison de sa gravité — avec, dans le cas de la religion romaine, le pouvoir d'en conjurer les effets ou de les expier par anti-

la garde constante des prêtres vigilants et n'était ouvert que lorsque de graves prodiges révélaient la colère des dieux contre Rome ". Raymond BLOCH, "La divination en Étrurie et à Rome » dans : André CAQUOT (dir.), La divination, T. 1, Paris, PUF, 1968, p. 215.

29. "Le Romain, lui, est superstitieux, sa culture scientifique reste longtemps limitée, et, de tous côtés, il voit tomber autour de lui la pluie des avertissements divins. Le ciel, la terre, le monde des plantes, des animaux et des hommes tours à tours présentent des phénomènes qui échappent selon lui aux lois naturelles et qu'il classe dans les rangs des prodiges : foudres, tremblements de terre, inondations, pluie insolite (...) ». Raymond BLOCH, "La divination en Étrurie et à Rome» dans : (André Caquot Dir.), La divination, T. 1, Paris, PUF, 1968, p. 224.

30. Roger CAILlOIS, Cohérences aventureuses, Paris, Gallimard, 1976, $281 \mathrm{p}$.

31. "Tandis que le présage avertit l'homme, de poursuivre ou d'arrêter sa route, le prodige révèle, pour sa part, que la paix des dieux est rompue et que la cité est gravement menacée par le courroux divin. (...) Le prodige est funeste et réclame une expiation rapide ". Raymond $\mathrm{BLOCH}$, "La divination en Étrurie et à Rome »dans : André CAQUOT (dir.), La divination, T. 1, Paris, PUF, 1968, p. 223-224. 
cipation. Dans l'optique de cette pensée magique, effacer l'hirondelle suffit à nous débarrasser du printemps.

Il importe avant tout dans cet horizon religieux de reconnaître le signe, le mauvais augure et d'y répondre en toute déférence, par la procédure exacte, pour interrompre sa venue ou faire en sorte qu'il ne s'accomplisse pas. Il faut simplement arrêter la fulmination procédurale des divinités. Les dieux sont en quelque sorte liés et assujettis par la réponse rituelle des défendeurs et leurs courroux atténués par la réponse et l'utilisation par l'homme des moyens formalistes pour y remédier. Religion et droit profane s'entrecroisent dans la haute précision des rites, si l'on considère justement qu'à l'instar de l'augure, le rite s'applique de la même manière que la scansion procédurale : "Le droit, en effet, tout comme la religion, ne pouvait être exercé qu'à l'aide du vocabulaire exact prévu par les «Actions de la Loi » qui ignoraient l'approximation et l'équivalence. À lui seul, l'énoncé exact faisait gagner le procès, à elle seule, la faute de langue le faisait perdre $» .^{32}$

\section{Examen et traitement des signes litigieux}

C'est que la religio désigne ici l'ensemble des liens juridiques qui unissent les dieux à Rome et une sorte de fair-play préside à ces échanges de bon procédés entre les parties. Comme le fait remarquer Jean Bayet dans son Histoire politique et psychologique de la religion romaine :

(...) pour les prodiges (naissance de monstres, pluie de pierres, etc...) ou pour les foudres - pour lesquelles la doctrine étrusque était très complexe, admettant des atténuations ou des remises, une sorte de domestication de l'éclair : les romains en cherchent avant tout la «liquidation ", ce qu'il appelle procuratio (conjuration anticipée), sans égard au contexte cosmique. Il leur suffit de rétablir pour eux-mêmes la paix des dieux, soit en supprimant le signe néfaste (enterrement de la foudre, noyade de l'androgyne), soit en se précautionnant de vieux procédés magiques (comme des ceps blancs ou le crâne d'un âne, qui mettent un champ, par exoratio, à l'abri de la foudre), soit par des rites soigneusement minutés.

32. Danielle PORTE, Les donneurs de sacré. Le prêtre à Rome, Paris, Éditions les Belles Lettres, 1989, p. 140. 
(...) La notion d'obligations juridiques réciproques (fides) en dérivait :

l'exactitude du rite entraîne l'acquiescement du dieu. ${ }^{33}$

L'attention divinatoire paraît à certains égards, dans son herméneutique fragile, l'ancêtre lointain du diagnostic savant, le premier essai d'une lecture rigoureuse, d'une exégèse prophylactique ${ }^{34}$ dans la délimitation d'un périmètre de sens strict. En fait, l'on sent bien aussi qu'il existe une communauté lointaine d'intérêts à l'égard de l'élément suspect, une " cohérence aventureuse " entre le praticien moderne penché sur l'image floue, la résonance magnétique d'un calcul rénal ou encore sur l'empreinte de la plus inquiétante micro-calcification mammaire et celui qui, dans la religion romaine, observait scrupuleusement les foies d'oiseaux (hépatoscopie), l'haruspice penché sur un fouillis d'entrailles.

Une même certitude dans le prononcé du diagnostic à la vue du hiéroglyphe précurseur, du polype irrégulier à distinguer du kyste bénin ou encore d'un caillot causé par une semence au jéjunum du volatile présageant un embâcle sur le Tibre et le faisant sortir de son lit. Le prêtre doit opérer une synthèse des signes mis à découvert dans un espace circonscrit. Cette attention discriminatoire constitue la première saisie scientifique, l'initiale observation des phénomènes derrière lesquels l'on présume - grâce à une série de figures limitées l'existence d'une volonté des dieux et plus tard celle de lois de la nature. Ce geste désormais tenu pour suspect fonde ainsi pourtant l'autorité du savoir par rapport à celui de la simple force ou de la contrainte.

Une pensée rationnelle s'emploie à faire correspondre comme sur les dents d'un engrenage exact, le ciel et la terre, dans la procédure très précise des rites qui enchâsse alors l'action humaine dans une sorte de bien-fondé cosmique. Rien n'est entrepris sans l'assurance de la collaboration divine. Cependant chacun des fonctionnaires de ces mantiques agit sa "spécialité » dans une relative indépendance à l'égard du travail des autres. Comme si tous ces espaces de lectures fonctionnaient en vase clos, sans mitoyennetés, pour minimiser à leur manière, toutes

33. Jean BAYET, Histoire politique et psychologique de la religion romaine, Paris, Payot, 1969 , p. 59.

34. Voir à ce propos la division précautionneuse de la parole de chacun des interprètes à la note 36 . 
les formes du changement et du devenir. Et pourtant dans l'esprit romain, on note une même observance scrupuleuse de la limite, une divinisation de ce qui borde et ne saurait souffrir quelque franchissement ${ }^{35}$. Augures et Haruspices opèrent de concert avec les magistrats et au terme de leurs inspections, un délicat partage de l'autorité politique. $^{36}$

\section{L’autorité de la parole}

Plus encore que la question de la validité ou la fausseté de cette science de l'interprétation, l'instauration d'un espace dogmatique qui répond à l'être humain demeure le fondement de cette politique augurale. C'est de la magie conjuratoire de la parole, du pouvoir, de l'autorité, qui se joue à travers tous les arrangements insolites des augures et des haruspices. L'évolution de cette science du vol ou de l'examen du foie d'oiseaux laissera place à la liberté de l'opérateur, du dessinateur du templum. Jean Bayet fait remarquer que :

(...) Ces techniciens eux-mêmes, fidèles à la tendance de l'esprit latin, se rendirent de plus en plus maîtres des signes qu'ils étaient censés subir. L'auspiciant développe encore les libertés traditionnelles : de son bâton

35. «La pierre qui fixait la limite du terrain était sacrée. (...) À la fête des Terminalia - le 23 février - le terminus recevait, chaque année, l'hommage du sacrifice qui répétait la cérémonie initiale. (...) L'État romain honorait également d'un culte la divinité qui protégeait la stabilité des limites agraires. (...) La vénération des bornes était vivante dans le peuple jusqu'à la fin de l'antiquité ». V. BASANOFF, Les dieux des romains, Paris, PUF, 1942, p. 52. 36. "L'observation des oiseaux ou des entrailles était faite, officiellement, par le magistrat muni du "droit d'auspices » : c'est donc le magistrat qui « observe " c'est-à-dire reçoit les signes que les dieux destinent à sa personne : tandis que l'Augure, lui, n'a que le droit de déclarer les signes reçus, après les avoir interprétés selon sa compétence technique : ainsi, le magistrat reçoit les auspices qu'il ne sait pas interpréter, tandis que le prêtre a cette science, mais doit se borner à lire les signes célestes destinés à un autre! Le prêtre observe sans avoir le droit d'observer, le magistrat, qui a ce droit, ne peut pas lire ce qu'il observe (....) ». Danielle PORTE, Les donneurs de sacré. Le prêtre à Rome, Paris, Éditions les Belles Lettres, 1989, p. 168. Il s'agit bien sûr, par le biais de ces clivages ou de cette séparation des pouvoirs, de maintenir une impartialité procédurale et de ne pas produire une lecture intéressée des situations. 
incurvé (lituus) il délimite le templum où les présages seront valables, l'oriente de fait et par ses paroles, il choisit les oiseaux, deux à gauche, deux à droite, qu'il entend observer ; il peut toujours n'avoir pas égard à un signe en disant non consulto ; il peut même nommer, donc faire exister, une chose non vue. Le choix du moment (tempestas), la disjonction de l'observation et de la renuntiatio (formulation), la constatation d'erreurs (vitia) et la reprise sur nouvelles bases d'auspices fautifs accroissent la part de l'arbitraire. ${ }^{37}$

Ainsi Émile Benveniste, dans son important ouvrage sur Le vocabulaire des institutions indo-européennes, note la contiguïté étymologique étroite qui fixe l'augure, non pas tant à la scrupuleuse topographie du vol parcouru ou de la viscère dépliée, mais plutôt dans le prononcé ostentatoire de la parole autorisée. Augeo, c'est la parole surgie du dispositif de l'examen selon des règles canoniques de l'oiseau, mais surtout l'interposition d'une parole différenciée au terme de ce montage d'un lieu de vérité et de pouvoir. Le linguiste écrit :

En latin même, à côté de auctor, nous avons un ancien neutre masculinisé augur, le nom de l' "augure ", avec son dérivé augustus, qui forment un groupe distinct. On voit l'importance double de ce groupe de mots. Ils appartiennent à la sphère politique et à la sphère religieuse et se sont scindés en plusieurs sous-groupes : celui de angeo, celui de auctor, celui de augur. (...) A augeo, on s'accorde à rattacher aussi le terme religieux augur. C'est déjà le sentiment des Latins. Augur serait un ancien neutre qui aurait désigné d'abord la " promotion » accordée par les dieux à une entreprise et manifestée par un présage. Cela confirme que l'action de augere est d'origine divine. Toute parole prononcée avec l'autorité détermine un changement dans le monde, crée quelque chose ; cette qualité mystérieuse, c'est ce que augeo exprime, le pouvoir qui fait surgir les plantes, qui donne existence à une loi. ${ }^{38}$ (nous soulignons).

Jupiter, un des dieux tout-puissants de la triade (avec Mars et Quirinus), protecteur de la nation romaine, fait connaître, à cette cohorte de dévoués subalternes, à travers des signes tangibles, sa volonté paternelle. Augures, Haruspices et Flamines sont liés à lui comme un collège de cardinaux, de docteurs le sont encore à l'endroit du souve-

37. Jean BAYET, Histoire politique et psychologique de la religion romaine, Paris, Payot, 1969 , p. 55.

38. Émile BENVENISTE, Le vocabulaire des institutions indo européennes 2. pouvoir, droit, religion, Paris, Éditions de Minuit, 1968, p. 148. 
rain pontife catholique. La discipline divinatoire n'est que le pré-texte à l'investiture politique des porte-parole dans leurs fonctions rassurantes de gouvernants, même si au plan factuel, cette discipline paraît d'une déconcertante fragilité. Ils sont à l'égard du sort du monde romain, ce que l'intercession du Pape demeure quant à la toute-puissance de Dieu le Père : "il détourne la menace absolue du Père toutpuissant, en énonçant, sous forme d'interprétations, un attirail compliqué d'arrangements conjuratoires. ${ }^{39}$

Il s'agit en fait de fonder l'Autorité sur un ordre du monde référencié. Que l'on se soit progressivement éloigné des formes pittoresques de l'examen des pierres de foudre ou des entrailles d'oiseaux pour se jeter et surtout s'appuyer sur le sens véritable à donner à un texte, préalablement déclaré inspiré par Dieu, ne change pas grand chose à l'affaire. "Celui qui dit le sens parle d'autorité, son dire réfère à un autre, un au-delà de sa parole qui la garantit comme parole et cautionne sa prétention de vérité. (...) Il y aurait, au sein de toute interprétation, une dimension "sacerdotale ", quelque chose de numineux. ${ }^{40}$

Cette discipline de lecture antique marque en Occident l'instauration d'un espace dogmatique, la formation d'une scène exégétique primordiale. "Le paradigme de la science augurale éclaire l'avènement du lieu séparé, l'autonomie de l'espace tiers dans la culture (...) ». ${ }^{41}$ Ces catégories de personnages attentifs à l'expression erratique des dieux - en communication avec eux par l'office des oiseaux — instituent les premiers jalons de la normativité sociale en sollicitant du tiers fictif une parole infaillible et en s'y soumettant. La prise des augures implique alors l'autorité de la question tranchée, de la chose jugée.

39. Pierre LEGENDRE, L'amour du censeur. Essai sur l'ordre dogmatique, Paris, Seuil, 1974, p. 105.

40. Jean FLORENCE, "Discours psychanalytique et discours juridique : remarques sur l'interprétation ", dans Michel van de KERCHOVE (dir.), L'interprétation en droit, approche multidisciplinaire, Bruxelles, Éditions des Facultés universitaires Saint-Louis, 1978, p. 551.

41. Pierre LEGENDRE, Leçons 1 La $901^{e}$ conclusion. Étude sur le théâtre de la Raison, Paris, Arthème Fayard, 1998, p. 300. 


\section{Divination et modernité}

Les pratiques de la religion romaine pourraient paraître superstitieuses. Pourtant ce que la religion gagne en clarté en abandonnant la divination, elle le perd en profondeur, jusqu'à s'affadir complètement dans des généralités morales, devenir parfaitement soluble dans la rationalité. D'autant que le souci rituel —dans sa prétention d'opérativité ou d'efficacité - procède comme par « enchantement verbal » de la mêmes solennité divinatoire et doit correspondre lui aussi, pour être valide, à ce que les linguistes appellent les conditions de " félicité linguistique ».

L'intérêt divinatoire participe surtout du désir irrépressible d'obtenir une réponse dans un univers compris comme une prodigieuse machine à produire à l'intérieur de ses usagers, toutes les formes de la déception, dont la dernière implique rien de moins que la disparition de l'écran sur lequel a lieu chacun de nos rêves et finalement de nousmême comme rêve. Dans ce dernier cas, personne ne se lève ni les lumières ne se rallument pour sortir de cet intime cinéma.

La recherche d'une cohérence ultime entre ses aspirations profondes et la réalité extérieure n'a rien d'une démarche initiatique. Elle n'a pas non plus à voir dans sa forme extrême avec l'hallucination mais ne se désespère pas de trouver, aux confins des solitudes, une solution à l'étrangeté de soi. À cet égard, il conviendrait peut-être de cesser de désigner dédaigneusement par l'expression " pensée magique » ce qui n'est en fait, que la forme authentique ou la plus sincère manifestation de notre détresse véritable. La facticité des professionnels de la pensée tient à la tenue d'un discours dont l'extériorité thématique ne saisit plus rien de l'enjeu véritable relié au fait de penser. Quiconque a conscience de vieillir comprend la nécessité absolue de la pensée magique et éventuellement des stupéfiants.

Il faut savoir rire des lucidités viriles capables de penser la mort : l'être humain n'a jamais su faire autre chose que de se payer de mots, d'établir en contrepartie du pire, toutes sortes de fausses connexions langagières, en un mot d'espérer. Et c'est là une des caractéristiques constitutives du langage que de s'ouvrir à la prédiction, que de se prêter à toutes les formes de l'expectative.

Divination et superstition sont les jumeaux intrigants d'une même compréhension poétique, celle de David devant ce Goliath de la réa- 
lité exacte, des projections chiffrées, des courbes actuarielles ou de l'encerclement croissant produit par le meilleur des mondes de la planification. L'espérance mathématique ne fait pourtant pas vivre.

L'acte divinatoire a, quant à son désir exorbitant de donner du sens et surtout d'en trouver dans l'univers, partie liée avec le phénomène religieux. Il implique de poser des gestes précis et de lire des conjonctures pour en extraire et en expliquer à la singularité d'un sujet qui s'en inquiète, la portée véritable. Toute une herméneutique savante de l'inopiné, de l'aléatoire ou de l'impondérable vient réduire l'inconnu - comme on parle en orthopédie de la réduction d'une fracture osseuse - à la certitude de signes qui ne sauraient mentir. Il en va tout autant dans la religion que dans la divination du maniement d'un transfert, de la proposition à un sujet angoissé d'un savoir supposé certain, quant à l'avenir.

Celui qui s'y présente avec sa question y vient dans un autre état, au sortir d'une macération inquiète, bien disposé à faire se précipiter ou coaguler pour lui-même, des liens suggérés entre des séries de signes et des événements. Une trame d'indices est ainsi arrachée à toutes contingences comme à tout hasard. Rien d'étonnant à ce que la religion chrétienne, ait très tôt senti le besoin de faire disparaître ce double gênant ou ce désagréable concurrent quant à l'exercice du contrôle monopolistique d'un sens commun ou de la compréhension ultime des fins dernières du genre humain. ${ }^{42}$

\section{L'écran de sauvetage}

Il ne se passe pas une soirée sans qu'un téléspectateur nord-américain ne soit désormais sollicité aux confins des émissions régulières, en fin de nuit, par un système commercial de prédictions astrologiques ou de diseurs de bonne aventure. Il semble que l'avenir individuel se donne à comprendre immédiatement après la vente des rôtissoires et autres babioles de l'info-pub et les lignes téléphoniques à contenus

42. «C’est un péché mortel de consulter les diseurs de bonne aventure, tireurs de cartes, chiromanciens, bohémiens ou tsiganes, si réellement on croit à la véracité de leurs prédictions ». T. ORTOLAN, "Divination " dans : A. VACANT (dir.) Dictionnaire de Théologie catholique (DTC), T. 4, Paris, Letouzey et Aney Éditeurs, 1911, p. 1453. 
érotiques. On peut ainsi imaginer toute une classe de la population non requise par un emploi matinal s'accrocher tantôt à l'internet, tantôt à ces dispositifs de la parole oraculaire partagée dans l'anonymat.

Cette manière nouvelle d'échanger porte à penser que les êtres humains aiment à marcher sur des fils tendus, à suivre le fil de conversations en trompe-l'œil, à communiquer à partir de leur univers intime dérisoire. Ces réseaux de communication tressés en filet empêchent ces funambules éphémères d'être aspirés par l'étrange dépressurisation des solitudes urbaines abyssales. Ce qui importe ici à l'usager c'est qu'il y ait quelqu'un au bout du fil, seulement une voix, quelqu'un qui sait, n'importe qui, une autre personne à qui parler ou écrire.

Il n'importe surtout pas ici de communiquer avec autrui comme visage mais de tromper sa solitude comme l'on trompe sa faim. L'inconnu au bout de la ligne n'est qu'un moyen de faire vibrer cet esseulement. Manière de dire, je ne veux pas tant te parler que de cesser de subir l'impression oppressante que je suis entièrement seul.

Ces systèmes de solidarité fictive (est-ce un pléonasme?) troublent par leur caractère rudimentaire à l'ère des diagnostics prénataux, des simulations actuarielles ou des bulletins météorologiques par satellite, parce qu'ils font ressortir un effort séculaire de mitigation du nonsens, une espérance individuelle de pacotille, non plus soutenue par la stabilité d'un cadre religieux balisé mais voguant plutôt à la recherche anxieuse de bouées solubles.

La désaffection de la religion en Occident entraîne à sa suite toutes les formes curieuses et fébriles de l'apesanteur destinale individuelle. Chacun cherche à connaître le tour que prendra l'écoulement d'une vie de consommateur. Elle constitue en fait une entreprise de prêt sur gage de sens, de prêt à porter pour affronter le malaise présent. Ces mêmes expédients désuets subsistent pourtant un peu de la manière du «pawn schop » qui coexiste avec la banque informatisée et tous ces systèmes de transactions virtuelles.

Une industrie molle de l'élucidation naïve (certain dirait de l'intoxication volontaire) de l'angoisse - sans doute moins lucrative que celle pharmaceutique de l'anxiolytique -, s'installe par voie publicitaire sur la dernière plage horaire, à la dernière frontière, avant que les écrans ne se confondent parfaitement avec la nuit. Il en va en fait 
du partage de l'angoisse autour de la question adressée à un inconnu, de la réponse vague censée faire la lumière sur le sentiment vécu par un des interlocuteurs : l'enfant pauvre de la psychanalyse. Il serait malheureux de ne réduire - en s'interdisant de le comprendre - cet exercice de communication qu'à une entreprise d'abus de la crédulité ou de la confiance des participants. Il met pourtant en lumière de la manière la plus crue des abîmes d'imperceptibles solitudes.

\section{RÉSUMÉ}

Dans ce bref essai, nous cherchons à surprendre la poésie d'une folie classificatoire qui se révèle à travers les subdivisions spécialisées des «métiers d'avenir » d'obédiences jupitériennes, des collèges de devins de l'ancienne Rome : Augures, Haruspices, Flamines, toutes espèces de techniciens des foudres et des viscères d'oiseaux affichant une inégalable componction dans l'exercice de leur art.

L'acte divinatoire vise à donner du sens et surtout à en trouver dans l'univers, partie liée avec le phénomène religieux. Toute une herméneutique savante de l'inopiné, de l'aléatoire ou de l'impondérable vient réduire l'inconnu à la certitude de signes qui ne sauraient mentir. Il en va tout autant dans la religion que dans la divination du maniement d'un transfert, de la proposition à un sujet angoissé d'un savoir supposé certain, quant à l'avenir.

\section{ABSTRACT}

In this brief essay we try to catch the poetic movement from a classified madness that is revealed through the specialized subdivisions of « métiers d'avenir » from Jupiter obediences, college of fortune tellers from ancient Rome: Augurs, Haruspices, Flamines, all sorts of technicians of lightning and birds viscera showing an incomparable compunction in their exercise of art.

The purpose of divine act is to give a signification and specially to find it inside the universe which is link to the religious phenomenon. An entirely part of unexpected, unpredictable, or imponderable scholarly hermeneutic reduces the unknown to the certainty of signs that could not have died. It is the same in religion as in the divine handling of transfering, in the proposition to an anxious subject of a supposely knowledge regarding the future. 